\title{
X-Ray Tomographic Imaging of Crystal Structure at the Atomic Level
}

\author{
P. Korecki, ${ }^{1, *}$ M. Tolkiehn, ${ }^{2}$ D. V. Novikov, ${ }^{2}$ G. Materlik, ${ }^{3}$ and M. Szymonski ${ }^{1}$ \\ ${ }^{1}$ M. Smoluchowski Institute of Physics, Jagiellonian University, Reymonta 4, 30-059 Kraków, Poland \\ ${ }^{2}$ HASYLAB at DESY, Notkestrasse 85, 22603 Hamburg, Germany \\ ${ }^{3}$ Diamond Light Source Limited, Chilton, Didcot Oxfordshire OX11 ODE, United Kingdom
}

(Received 1 August 2005; published 24 January 2006)

\begin{abstract}
A direct nondiffractive tomographic algorithm is proposed for the determination of the crystal structure from real-space projections obtained by illuminating the sample with white $\mathrm{x}$ rays. This approach was applied to the pattern of the directional fine structure in absorption of white $\mathrm{x}$ rays recorded for a $\mathrm{GaP}$ crystal and allowed for a determination of the electron density distribution within the unit cell.
\end{abstract}

DOI: $10.1103 /$ PhysRevLett.96.035502

The majority of x-ray methods for crystal structure investigations are based on diffraction phenomena and sample the information in the reciprocal space by measuring the intensities at discrete Bragg peak positions [1]. Usually, a Fourier transformation is used to recover the electron density distribution of the object. However, a direct back transform is hindered by the lack of phase information in the recorded intensity data and the inversion algorithms are ambiguous. The phase information can become directly accessible, e.g., by using anomalous [2] or multibeam x-ray diffraction [3]. Another approach involves measuring the $\mathrm{x}$-ray wave field intensity at atomic sites inside a crystal as it is performed in the $\mathrm{x}$-ray standing wave technique [4] or in $\mathrm{x}$-ray holography $(\mathrm{XH})[5,6]$. The absorption cross section in these methods is modulated by x-ray diffraction, which results in an angular dependent absorption fine structure measured through the secondary yield coming from specific sorts of atoms. This directional fine structure can be inverted to real space by using Fourier [7] or holographic reconstruction [8]. All these methods utilize monochromatic $\mathrm{x}$-ray radiation.

In this Letter we propose and demonstrate experimentally that crystal structure can be recovered from real-space projections obtained with polychromatic x rays (hereafter called white $\mathrm{x}$ rays), by using a nondiffractive tomographic algorithm. This approach is based on recent work [9], which showed that the directional absorption fine structure is related to the spherical projection of crystal structure. For a white $\mathrm{x}$-ray beam, the wave field, formed by interference of the incident beam with the waves scattered on single atoms, cancels out by energy integration for all directions, except for the forward scattering component, coinciding with the incident beam. In a practical case, the white $\mathrm{x}$-ray radiation is not uniform over the whole energy range, which leads to remnant high order diffraction effects. However, in the presence of long-range order, the distortion of the real-space projection is small for directions coinciding with the lattice planes and, as it will be shown, does not significantly influence tomographic reconstruction.

Scattering of spherical waves from atoms of the sample onto an absorbing atom results in directional absorption
PACS numbers: 61.10. $-\mathrm{i}, 42.30 . \mathrm{Rx}$, 81.70.Tx

fine structure [10]. For a white $\mathrm{x}$-ray beam this fine structure can be written as [9]

$$
\chi(\hat{\mathbf{k}})=-2 r_{0} \operatorname{Re} \int_{0}^{\infty} N(k) \int_{V_{s}} \rho(\mathbf{r}) \frac{e^{i k r}}{r} e^{i \mathbf{k} \cdot \mathbf{r}} d V d k,
$$

where $\hat{\mathbf{k}}$ is the beam direction, $r_{0}$ is the Thomson scattering length, $N(k)$ is the effective x-ray spectrum, $\rho(\mathbf{r})$ is the electron density, $\mathbf{r}$ is the position relative to the absorbing atom, and the integral extends over the volume $V_{s}$ of the sample. For a perfect white beam, i.e., for $N(k)=1$, it was shown that [9]

$$
\begin{aligned}
\chi(\hat{\mathbf{k}}) & =-2 \pi r_{0} \int_{V_{s}} \frac{\rho(\mathbf{r})}{r} \delta(r+\hat{\mathbf{k}} \cdot \mathbf{r}) d V \\
& =-2 \pi^{2} r_{0} \int_{0}^{\infty} \rho(-\hat{\mathbf{k}} r) d r
\end{aligned}
$$

In Eq. (2), $\chi(\hat{\mathbf{k}})$ can be interpreted as a projection of $\rho(\mathbf{r})$ onto a sphere. In the general case a reconstruction of $\rho(\mathbf{r})$ from $\chi(\hat{\mathbf{k}})$ is impossible. However, one can take advantage of the periodicity of $\rho(\mathbf{r})$ and write it as $\rho(\mathbf{r})=$ $V^{-1} s(\mathbf{r}) \sum_{\mathbf{H}} F_{\mathbf{H}} e^{i \mathbf{H} \cdot \mathbf{r}}$, where $s(\mathbf{r})$ is the shape function [1], which for simplicity is assumed to have spherical symmetry, and $V$ is the unit cell volume. Thus, for negligible dispersion correction of x-ray scattering (i.e., for $F_{-\mathbf{H}}=$ $F_{\mathbf{H}}^{*}$ ) Eq. (2) can be written as

$$
\chi(\hat{\mathbf{k}})=-\frac{2 \pi^{2} r_{0}}{V} \sum_{\mathbf{H}}\left[\operatorname{Re}\left(F_{\mathbf{H}}\right) \chi_{\mathbf{H}}^{+}-\operatorname{Im}\left(F_{\mathbf{H}}\right) \chi_{\mathbf{H}}^{-}\right],
$$

where

$$
\begin{gathered}
\chi_{\mathbf{H}}^{+}(\hat{\mathbf{k}})=\pi \delta(\mathbf{H} \cdot \hat{\mathbf{k}}) * S(\mathbf{H} \cdot \hat{\mathbf{k}}), \\
\chi_{\mathbf{H}}^{-}(\hat{\mathbf{k}})=\frac{1}{\mathbf{H} \cdot \hat{\mathbf{k}}} * S(\mathbf{H} \cdot \hat{\mathbf{k}}),
\end{gathered}
$$

and $S(\mathbf{H} \cdot \hat{\mathbf{k}})$ is the Fourier transform of the onedimensional central cross section of $s(\mathbf{r})$. These equations describe the directional signal as being composed of discrete bands localized along projections of lattice planes, i.e., on great circles on the sphere. These bands are linear functions of the structure factors $F_{\mathbf{H}}$. Each band results 
from a superposition of terms corresponding to the set of all collinear reciprocal lattice vectors $\mathbf{H}$. Depending on the position of the absorbing atom relative to the planes forming the band, the band can be symmetric or asymmetric. $S(\mathbf{H} \cdot \hat{\mathbf{k}})$ is a function sharply peaked at zero, and its spread is inversely proportional to the size of the sample. Thus, for sufficiently large samples, the strongest bands are well separated and a significant overlap between them occurs only at crossing points.

For a nonuniform white spectrum $N(k)$, the remnant diffraction will distort the functions $\chi_{\mathbf{\mathbf { H }}}^{ \pm}$. In a practical case, $N(k)$ can often be approximated with a Lorentzian. This allows us to calculate the $\chi_{\mathbf{H}}^{ \pm}$analytically. The calculation is straightforward but cumbersome and will be published elsewhere. Examples of bands calculated for parameters, corresponding to those used in the experiment, are shown in Fig. 1(a) for different positions of absorbing atoms relative to the lattice planes. These bands have a characteristic half-width given by $\Delta \vartheta_{\hat{\mathbf{n}}}=$ $\arcsin \left[2 \pi /\left(d_{\hat{\mathbf{n}}} \sqrt{4 k_{0}^{2}+\Delta k^{2}}\right)\right]$, where $k_{0}$ and $\Delta k$ are wavevector equivalents of the mean energy and the energy spread and $d_{\hat{\mathbf{n}}}$ is lattice plane spacing corresponding to the first order reciprocal vector parallel to $\hat{\mathbf{n}}$. For $\Delta k=0$, this expression reduces to the Bragg condition $\lambda=$ $2 d_{\hat{\mathbf{n}}} \sin \Delta \vartheta_{\hat{\mathbf{n}}}$.
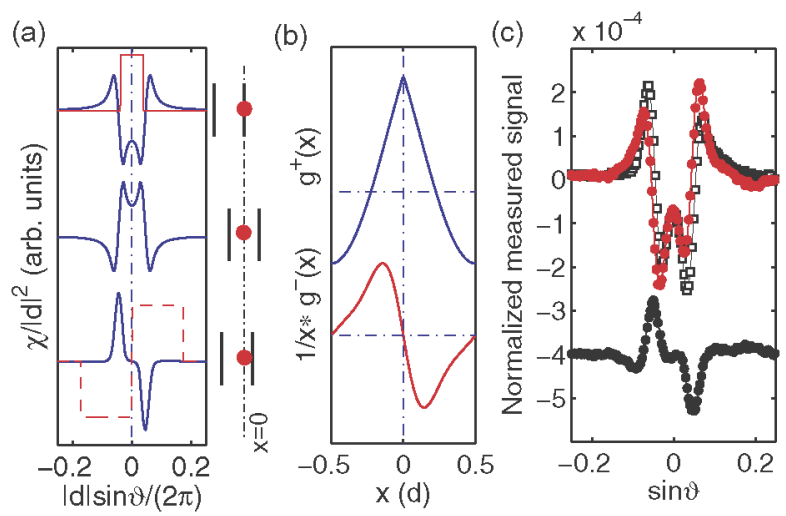

FIG. 1 (color online). Calculated and measured band profiles in the directional fine structure of white $\mathrm{x}$-ray absorption. (a) Shape of bands calculated using a realistic energy spectrum for absorbing atoms placed at different positions relative to lattice planes. The positions are shown next to the plots and $d$ is the lattice plane spacing. The two upper plots show the two possible symmetrical configurations, whereas the bottom plot shows an asymmetric one. The window functions $w^{+}$(solid line) and $w^{-}$(dashed line) used for data evaluation are superimposed on the model curves. (b) Functions arising in the projection integrals calculated for bands shown in (a): $g^{+}(x)$ (top) and $1 / x * g^{-}(x)$ (bottom). Dash-dotted lines show zero levels. (c) Top curve: Measured integrated intensity of (111) band (solid circles, GaP A side; open squares, GaP B side). Bottom: the difference of the two experimental curves.
Let us now prove that the directional white $\mathrm{x}$-ray absorption fine structure can be tomographically reconstructed to recover the electron density. Consider a single intensity band, determined by the unit vector $\hat{\mathbf{n}}$ normal to the corresponding set of lattice planes and two quantities measured across the band from the experimental pattern $\chi(\hat{\mathbf{k}})$ :

$$
I_{\hat{\mathbf{n}}}^{ \pm}=\int w^{ \pm}\left(\vartheta_{\hat{\mathbf{n}}}\right) \chi\left(\vartheta_{\hat{\mathbf{n}}}\right) d \vartheta_{\hat{\mathbf{n}}},
$$

where $I_{\hat{\mathbf{n}}}^{+}$measures the intensity near the center of the band and $I_{\hat{\mathbf{n}}}^{-}$its asymmetry. The angle $\vartheta_{\hat{\mathbf{n}}}$ is the deviation from the center of the band, and $w^{+}\left(\vartheta_{\hat{\mathbf{n}}}\right)$ and $w^{-}\left(\vartheta_{\hat{\mathbf{n}}}\right)$ are normalized even and odd rectangular window functions [11]. The windows are introduced to remove effects caused by overlapping of bands [see Fig. 1(a)]. For further reduction of the interferences with other bands, these quantities should be averaged along the great circle. From Eqs. (3) and (6) it follows that

$$
\begin{gathered}
I_{\hat{\mathbf{n}}}^{+}=-\frac{4 \pi^{2} r_{0}}{V} \sum_{\mathbf{H} \| \hat{\mathbf{n}}} \operatorname{Re}\left(F_{\mathbf{H}}\right) G^{+}(H), \\
I_{\hat{\mathbf{n}}}^{-}=\frac{4 \pi^{2} r_{0}}{V} \sum_{\mathbf{H} \| \hat{\mathbf{n}}} \operatorname{Im}\left(F_{\mathbf{H}}\right) G^{-}(H),
\end{gathered}
$$

where the summation is performed only for $\mathbf{H} \cdot \hat{\mathbf{n}}>0$ and

$$
G^{ \pm}(H)=\int w^{ \pm}(\vartheta) \chi_{H}^{ \pm}(\vartheta) d \vartheta
$$

depend only on the length $H$ of $\mathbf{H}$. Using Plancherel's theorem and the identity $1 / x * \cos (n x)=\pi \sin (n x)$, based on Eqs. (7) and (8), one can define

$$
\begin{array}{r}
I_{\hat{\mathbf{n}}}^{+}\left(m d_{\hat{\mathbf{n}}}\right) \propto-\int\left[\rho(\mathbf{r}) * g^{+}(\hat{\mathbf{n}} \cdot \mathbf{r})\right] \delta\left(m d_{\hat{\mathbf{n}}}-\hat{\mathbf{n}} \cdot \mathbf{r}\right) d V, \\
I_{\hat{\mathbf{n}}}^{-}\left(m d_{\hat{\mathbf{n}}}\right) \propto-\int\left[\rho(\mathbf{r}) * g^{-}(\hat{\mathbf{n}} \cdot \mathbf{r})\right] \frac{1}{m d_{\hat{\mathbf{n}}}-\hat{\mathbf{n}} \cdot \mathbf{r}} d V,
\end{array}
$$

where the convolution is performed along the $\hat{\mathbf{n}}$ axis and $g^{ \pm}(r)=\sum_{\mathbf{H} \| \hat{\mathbf{n}}} G^{ \pm}(H) \cos (H r)$ are even functions, which for realistic $s(\mathbf{r})$ peak at zero. For $m=0$, Eqs. (10) and (11) are equivalent to Eqs. (7) and (8), respectively. However, one can use the invariance of integrals in Eqs. (10) and (11) with respect to translation in the direction of $\hat{\mathbf{n}}$ by $d_{\hat{\mathbf{n}}}$ and write them for any integer $m$. Note that, for a sufficiently broad $N(k)$ spectrum, the general form of Eqs. (3)-(11) will not change. The shape of $N(k)$ influences only the exact form of the functions $g^{ \pm}$. These functions calculated for parameters corresponding to experimental situations are shown in Fig. 1(b).

Equations (10) and (11) can be used as a basis for the tomographic reconstruction algorithm. In tomography the object is reconstructed from one-dimensional projections 
obtained at different illumination angles [12]. For a description of a point source broadband tomographic geometry, which is closely related to the present work, see $[13,14]$. The projection $p_{\hat{\mathbf{n}}}(d)$ of a three-dimensional object $\rho(\mathbf{r})$ can be described by the 3D Radon transform, i.e., by the plane integral [15]:

$$
p_{\hat{\mathbf{n}}}(d)=\int \rho(\mathbf{r}) \delta(d-\hat{\mathbf{n}} \cdot \mathbf{r}) d V
$$

where $\hat{\mathbf{n}}$ is normal to the integration plane and $d$ is a scalar defining the plane offset. Comparison of Eq. (10) with Eq. (12) shows that $I_{\hat{\mathbf{n}}}^{+}\left(m d_{\hat{\mathbf{n}}}\right)$ is analogous to a 3D Radon transform of the low-pass filtered electron density distribution $\rho(\mathbf{r}) * g^{+}(\hat{\mathbf{n}} \cdot \mathbf{r})$. The projections are sampled for a discrete number of illumination angles $\hat{\mathbf{n}}$ and virtually for a discrete number of plane offsets $m d_{\hat{\mathbf{n}}}$. Similarly, Eq. (11) can be interpreted as the 3D Radon transform of the lowpass filtered asymmetry of the electron density distribution. The width of the $g^{ \pm}$functions is of the order of $\sim d_{\hat{\mathbf{n}}} / 2$. This ensures that the data are appropriately sampled and no significant aliasing will occur [12], provided that both $I^{ \pm}$ are used for reconstruction. Therefore it becomes possible to reconstruct the atomic structure of the periodic object without a priori information by using tomographic reconstruction procedures [12,15].

To check the tomographic algorithm, we performed an experiment for a GaP crystal, which crystallizes in the $\mathrm{ZnS}$ structure $\left(a_{0}=5.45 \AA\right)$, and has no center of inversion. Two single crystal GaP wafers, with orientations (111) (A side) and (111) (B side), respectively, were used in the experiment. The white $\mathrm{x}$-ray synchrotron beam $(0.5 \times$ $0.5 \mathrm{~mm}^{2}$ ) was produced by a bending magnet at HASYLAB/DESY. A $10 \mathrm{~mm}$ thick $\mathrm{Al}$ absorber was used to shape a white effective energy spectrum having mean energy $E_{0} \sim 40 \mathrm{keV}$ and energy spread $\Delta E \sim 10 \mathrm{keV}$. The effective spectrum is determined by the synchrotron source emission characteristics, transmission of all elements placed in the beam, and the energy dependence of the absorption in the sample. The beam intensity was monitored with a photodiode at an average current of $\sim 10 \mu \mathrm{A}$. To probe the absorption, the sample, biased to $-90 \mathrm{~V}$, was placed inside a gas flow total electron yield detector filled with $\mathrm{He}$. The measured sample currents were in the range of $10 \mathrm{nA}$, whereas the direction dependent fine structure of the signal stayed at the level of $10 \mathrm{pA}$. The x-ray absorption of $\mathrm{Ga}$ atoms is about an order of magnitude higher than that of $\mathrm{P}$ atoms. The secondary electron yield is therefore dominated by the $\mathrm{Ga}$ atoms contribution, and this provides the element sensitivity of the measurements. For measuring the directional dependence of white x-ray absorption, the electron yield was collected while the sample was rotated relative to the beam direction. The rotation around the sample normal was performed continuously with an integration step of $0.3^{\circ}$. The inclination angle was changed step-by-step with $0.5^{\circ}$ intervals. The acquisition of a full pattern took $\sim 24 \mathrm{~h}$. For evaluation, a slowly varying background was subtracted for each azimuthal scan separately. The patterns were symmetrized in accordance with the rotation axes and mirror planes. No filtering procedures were applied.

The measured directional absorption fine structure of white x-ray absorption is presented in Figs. 1(c) and 2(a). The main features of the signal are bands coinciding with the real-space projections of crystal planes as shown in the stereographic projection of the GaP crystal from Fig. 2(b). Some bands, in particular $\{111\}$, display a significant asymmetry. This asymmetry is especially visible in the integrated profiles and their difference recorded for $\mathrm{A}$ and $\mathrm{B}$ sides. The difference in signal recorded for $\mathrm{A}$ and $B$ sides arises from the noncentrosymmetric structure of $\mathrm{GaP}$ and contains only the information about the P sublattice. The asymmetric shape of bands is directly connected to a different offset of $\mathrm{P}$ planes relative to $\mathrm{Ga}$ atoms for the two crystal orientations. This allows for determi-

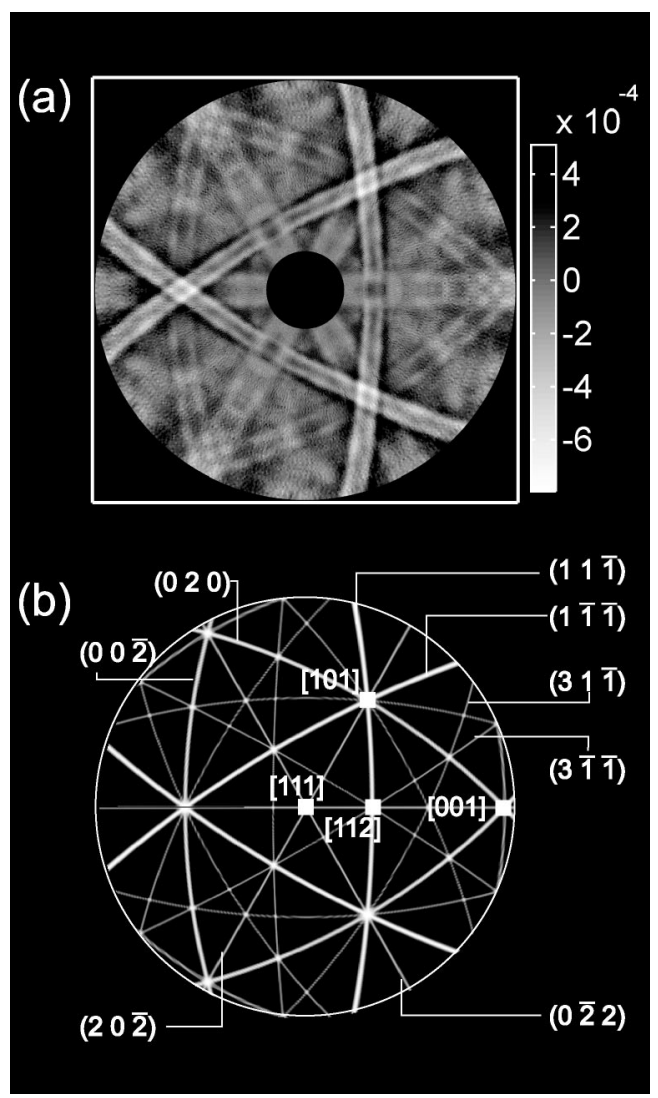

FIG. 2. Directional fine structure of white x-ray absorption recorded in the experiment. (a) Pattern recorded for $\mathrm{GaP}$ B sample. Each point in the pattern corresponds to a given orientation of the sample relative to the x-ray beam. The whole pattern is presented as a stereographic projection. The intensity is normalized to the slowly varying background. The visible bands correspond to real-space projections of the lattice planes. (b) Stereographic projection of the zinc blende structure. The lines are geometrical projections of lattice planes. 


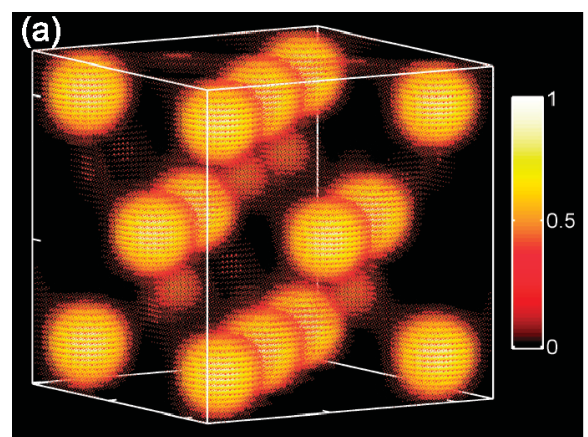

(b)

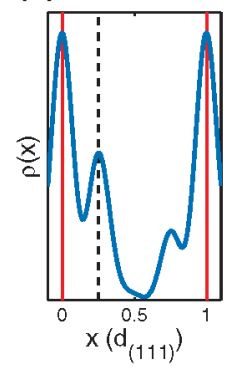

FIG. 3 (color). Electron density of GaP directly restored from experimental data with the tomographic algorithm. (a) Threedimensional view. Each voxel is represented as a small bubble with radius and color proportional to $\rho(\mathbf{r})$. The edges of the box are parallel to $\langle 001\rangle$ directions. The axis ticks are separated by $a_{0} / 2$. (b) Electron density profile along unit cell diagonal. Solid and dashed lines show the positions of $\mathrm{Ga}$ and $\mathrm{P}$ atoms, respectively.

nation of the polarity of the sample. Such an information is not accessible in the recently proposed methods of direct structure retrieval from $\mathrm{XH}$ patterns, which probe only the real parts of the structure factors, i.e., the symmetrical part of the structure [16]. Besides, in XH and in Kikuchi electron diffraction, the asymmetry of the signal can also be due to dynamical extinction effects and does not ultimately arise from structural differences $[17,18]$. Here, a direct comparison of the data, recorded in the same condition for both crystal orientations, shows that the asymmetry comes solely from structural effects.

The intensity and asymmetry were measured for 21 different bands, and these data were taken as input to the tomographic algorithm. The lacking data were taken from symmetry relations. The electron density distribution that was restored from the experimental pattern is shown in Fig. 3. The spatial resolution is limited by the lattice spacing of the analyzed bands having highest order, i.e., to $d_{(113)} / 2 \sim 0.8 \AA$. The additional small maximum in the profile shown in Fig. 3(b) is an image of Ga atoms as seen from $\mathrm{P}$ atoms. It results from a nonzero contribution of $\mathrm{P}$ atoms absorption to the total electron yield. It can be avoided by the detection of characteristic radiation. From the intensity of this artifact one can estimate the sensitivity of the method for detecting low- $Z$ elements at the level of at least 3 electrons per atom.

In summary, a direct tomographic algorithm for threedimensional imaging of a crystal structure with white $\mathrm{x}$ rays was proposed and tested in the experiment on a $\mathrm{GaP}$ single crystal. This algorithm takes advantage of kinematical scattering of $\mathrm{x}$ rays and permits a direct reconstruction of an atomic structure from real-space pro- jections. This is hardly possible from forward scattering patterns observed in electron diffraction [19]. The use of more powerful radiation sources will increase the photon flux by several orders of magnitude. More flux would allow one to detect characteristic radiation instead of total electron yield. In such a case, the tomographic algorithm could be applied to directly determine foreign atom positions in single crystals. Auxiliary simulations showed that the tomographic approach already works for small clusters having radii of $\sim 10 \mathrm{~nm}$. Therefore, experiments on lowdimensional systems, e.g., thin films or buried interfaces have in principle become possible. Because of the slow angular dependence of the measured signal, the systems under investigations could be highly imperfect.

This work was supported by Volkswagen Foundation, Federal Republic of Germany. The access to synchrotron was supported by the European Community-Research Infrastructure Action under the FP6 (Integrating Activity on Synchrotron and Free Electron Laser Science).

*Electronic address: korecki@if.uj.edu.pl

[1] J.M. Cowley, Diffraction Physics (North-Holland, Amsterdam, 1975).

[2] Resonant Anomalous X-Ray Scattering, edited by G. Materlik, C.J. Sparks, and K. Fischer (NorthHolland, Amsterdam, 1994).

[3] E. Weckert and K. Hummer, Acta Crystallogr. Sect. A 53, 108 (1997).

[4] M. J. Bedzyk and G. Materlik, Phys. Rev. B 32, 6456 (1985).

[5] M. Tegze and G. Faigel, Nature (London) 380, 49 (1996).

[6] T. Gog et al., Phys. Rev. Lett. 76, 3132 (1996).

[7] L. Cheng et al., Phys. Rev. Lett. 90, 255503 (2003).

[8] J. J. Barton, Phys. Rev. Lett. 61, 1356 (1988).

[9] P. Korecki and G. Materlik, Phys. Rev. Lett. 86, 2333 (2001).

[10] B. Adams et al., Phys. Rev. B 57, 7526 (1998).

[11] The rectangular window functions are defined as $w^{+}(\vartheta)=1 / \Delta w^{+} \quad$ for $\quad|\vartheta| \leq \Delta w^{+} / 2, \quad w^{-}(\vartheta)=$ $\operatorname{sgn} \vartheta / \Delta w^{-}$for $|\vartheta| \leq \Delta w^{-} / 2$, and 0 elsewhere.

[12] A. C. Kak and M. Slaney, Principles of Computerized Tomographic Imaging (IEEE Press, New York, 1988).

[13] R.P. Porter, Progress in Optics (North-Holland, Amsterdam, 1989), Vol. XXVII, p. 317.

[14] J. Rous and M. H. Rubin, Surf. Sci. 316, L1068 (1994).

[15] F. Natterer, The Mathematics of Computerized Tomography (Wiley, Chichester, 1986).

[16] S. Marchesini et al., Phys. Rev. B 66, 094111 (2002).

[17] P. Korecki et al., Phys. Rev. B 69, 184103 (2004).

[18] B. Fultz and J. M. Howe, Transmission Electron Microscopy and Diffractometry of Materials (Springer, Berlin, 2002).

[19] J. Osterwalder et al., Phys. Rev. B 44, 13764 (1991). 